Article

\title{
Highly Sensitive Detection of 4-Methylimidazole Using a Terahertz Metamaterial
}

\author{
Hee Jun Shin ${ }^{1}$, Hae Won Jang ${ }^{2} \mathbb{D}$ and Gyeongsik Ok ${ }^{3, *}$ \\ 1 Pohang Accelerator Laboratory, 80 Jigokro-127-beongil, Nam-Gu, Pohang, Gyeongbuk 37673, Korea; \\ shinhj@postech.ac.kr \\ 2 Research Group of Food Processing, Korea Food Research Institute, 245, Nongsaengmyeong-ro, Iseo-myeon, \\ Wanju-gun, Jeollabuk-do 55365, Korea; hwjkfri@kfri.re.kr \\ 3 Research Group of Consumer Safety, Korea Food Research Institute, 245, Nongsaengmyeong-ro, Iseo-myeon, \\ Wanju-gun, Jeollabuk-do 55365, Korea \\ * Correspondence: gsok@kfri.re.kr; Tel.: +82-63-219-9408
}

Received: 17 October 2018; Accepted: 4 December 2018; Published: 6 December 2018

check for updates

\begin{abstract}
In this study, we demonstrated a highly sensitive detection method of 4-methylimidazole (4-MeI), a carcinogenic material, by using a terahertz (THz) metamaterial at a $\mathrm{THz}$ region. The $\mathrm{THz}$ metamaterials were fabricated with a metal array, using an electric-field-coupled inductor-capacitor (ELC) resonator structure, and a finite-difference time-domain (FDTD) simulation showed good agreement with the experimental results. We measured the $\mathrm{THz}$ spectra of the metamaterials to detect the 4-MeI concentrations of $0,1,2,5,10,15$, and $20 \mathrm{mg} / \mathrm{L}$. The resonance frequency of the metamaterial was shifted by, approximately, $8 \mathrm{GHz}$ and transmittance at the resonance frequency increased to $2 \times 10^{-3}$, as the concentration was increased, up to $20 \mathrm{mg} / \mathrm{L}$. Our study provides new insight into the application of metamaterials in detecting carcinogens, using a $\mathrm{THz}$ technique.
\end{abstract}

Keywords: THz spectroscopy; 4-methylimidazole; metamaterial; carcinogen detection

\section{Introduction}

Several soft drinks, including sodas, energy drinks, and diet drinks, contain a caramel color which is widely used as a coloring and flavoring agent [1,2]. Caramel-colored drinks, including colas, root beer, and black tea, are widely consumed across the world in large amounts. However, caramel color agents have attracted significant attention because it was found that caramel-colored drinks may contain 4-methylimidazole (4-MeI), which is known to be carcinogenic [1,3-5]. Caramel colors are usually classified into four types, Caramel I, II, III, and IV, and their production and choice of reagents are based on their applications [2,3]. The compound 4-MeI is formed during the production of caramel colors type III and IV, which contain ammonium compounds. Detecting 4-MeI in food products has been an ongoing challenge, with several studies directed at developing efficient methods. For example, high performance liquid chromatography (HPLC) [6], HPLC-mass spectroscopy [3,7], gas chromatography (GC) [8], and GC-mass spectroscopy [9,10], have been used to detect 4-MeI, in addition to 2-dimensional liquid chromatography [11,12]. Spectral properties of various imidazole compounds and characteristics of imidazole isomers can be investigated using nuclear magnetic resonance and mass spectroscopy [13,14]. However, new detection techniques using label-free technologies, with high sensitivity and ease of use, have been in high demand, especially, in cases of chemical or biological material testing.

Following recent reports that terahertz $(\mathrm{THz})$ waves can be generated using a femtosecond laser, application of the $\mathrm{THz}$ frequency range has gained significant attention in various research areas, including biochemistry [15-18], electronics [19], photonics [20,21], astronomy [22], military 
science [23], food science [24,25], and medical diagnosis [26,27]. In particular, THz techniques such as THz time-domain spectroscopy, have emerged as attractive techniques that can enable label-free, non-contact, and non-destructive detection in biological and chemical materials $[18,28,29]$. The $\mathrm{THz}$ range can reveal several types of information in biological and chemical materials; for example, various conformational energies, such as rotation, vibration, and intermolecular interactions of chemical and biological molecules occur in the THz range $[18,24,30]$. Recent efforts to improve sensitivity of biochemical detection have involved the use of various artificial structures that are fabricated using metamaterials. Metamaterials have several significant advantages, including easy preparation, easy handling, high sensitivity, and material-selective procedures. Usually, artificial structures created using conductive materials, such as metals, are used for patterning metamaterials, and this provides an opportunity to control electromagnetic properties and functionalities of the materials. In addition, metamaterials have been designed to function in various frequency ranges, using their unique properties, including negative refractive index, and high absorbance at specific frequencies. In particular, $\mathrm{THz}$ metamaterials have been widely studied because they can be used to design artificial structures to enhance the detection of several chemical and biological materials in the $\mathrm{THz}$ range. $\mathrm{THz}$ metamaterials can be used to markedly enhance biochemical molecular energies in the THz region, and they have been investigated in various biological and chemical studies [31-39].

In the present study, we demonstrated the detection of 4-MeI, a possible carcinogenic component in caramel-colored drinks, using $\mathrm{THz}$ metamaterials. We measured the $\mathrm{THz}$ spectrum of 4-MeI, in the frequency range of 0.2 to $2 \mathrm{THz}$, using the $\mathrm{THz}$ transmission mode spectroscopy. Using a $\mathrm{THz}$ metamaterial at the resonance frequency of 4-MeI, we investigated its frequency shift enhancement for 4-MeI detection.

\section{Materials and Experiment}

\subsection{Metamaterial Fabrication}

The $\mathrm{THz}$ metamaterial was fabricated using an electric-field-coupled inductor-capacitor (ELC) resonator structure $[40,41]$. The metamaterial was based on a metallic array consisting of a square ring with a micro split gap structure. A polyimide (PI) solution was spin-coated onto the Si wafer, at a thickness of $500 \mu \mathrm{m}$, at $2500 \mathrm{rpm}$. The Si wafer with a coated PI solution was baked at $110^{\circ} \mathrm{C}$, for $3 \mathrm{~min}$. To deposit an Au pattern on the PI/Si wafer, we adopted a lift-off process. The PI/Si wafer substrate was photoresist-coated at $3000 \mathrm{rpm}$ for $40 \mathrm{~s}$ and soft baked at $95^{\circ} \mathrm{C}$ for $90 \mathrm{~s}$. Next, the substrate was exposed to UV for three seconds. Hard baking was performed at $110{ }^{\circ} \mathrm{C}$, for $3 \mathrm{~min}$, followed again by UV exposure for $30 \mathrm{~s}$. Finally, the photoresist was developed. We deposited a Ti $30 \mathrm{~nm} / \mathrm{Au} 100 \mathrm{~nm}$ layer on the PI/Si wafer. The schematic of the metamaterial is shown in Figure 1a. We simulated the metamaterial design using a finite-difference time-domain (FDTD) method, in the terahertz range.
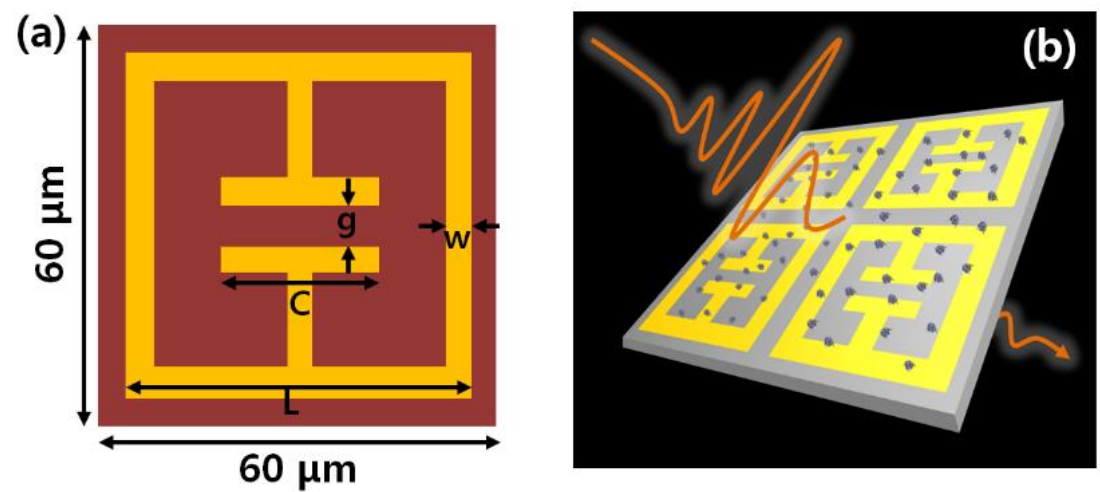

Figure 1. Schematic of (a) the terahertz (THz) metamaterial and (b) $\mathrm{THz}$ measurement, using the metamaterial. 
As can be seen in Figure 1b, a THz electric field passing through the metamaterial was scattered by the 4-MeI molecules in a vertical direction. The THz output signal throughout the metamaterial, was analyzed using a Fourier transformation-based method. A commercial THz time-domain spectroscopy system (TPS-3000, Teraview, Cambridge, UK) was used to obtain the THz spectra of the pellet sample and the metamaterial, in the frequency range of 0.2 to $2 \mathrm{THz}$. All of the experimental THz-TDS measurements were performed at room temperature and the humidity in the THz-TDS system was maintained at lower than $1 \%$, using dried air, during the $\mathrm{THz}$ spectra measurement of the samples.

The output $\mathrm{THz}$ signal $O(\omega)$, passing through a sample, is related to the input signal $I(\omega)$ as follows [18]

$$
O(\omega)=I(\omega) \exp \left[-\frac{d \alpha(\omega)}{2}\right] \exp \left[i \frac{2 \pi}{\lambda} n_{1}(\omega) d\right],
$$

where $I(\omega)$ is the complex input signal, $\alpha(\omega)$ is the absorption coefficient, $d$ is the thickness of the sample, $\lambda$ is the wavelength, and $n$ is the refractive index of the sample. Power absorption can be obtained from the difference between spectral amplitudes with and without the sample.

$$
\alpha(\omega)=-\frac{2}{d} \ln \left[\frac{O(\omega)}{I(\omega)}\right] .
$$

\subsection{4-MeI Sample Preparation}

A commercially available 4-MeI product was obtained (Sigma-Aldrich, St. Louis, MO, USA). We crushed the dry 4-MeI into small particles of sizes less than the THz wavelength, to prevent scattering. To measure the $\mathrm{THz}$ spectrum, the 4-MeI (30 mg) was prepared as a pellet sample compounded with a polyethylene $(\mathrm{PE})$ powder $(270 \mathrm{mg})$. In addition, we prepared 4-MeI solutions to apply onto the $\mathrm{THz}$ metamaterial, at concentrations of $0,1,2,5,10,15$, and $20 \mathrm{mg} / \mathrm{L}$ of $4-\mathrm{MeI}$, in distilled water. Ten microliter of the 4-MeI solutions, at each concentration, were dropped onto the $\mathrm{THz}$ metamaterial and dried at $70{ }^{\circ} \mathrm{C}$, for $10 \mathrm{~min}$, until the water evaporated completely. Next, the THz metamaterial was cooled, at room temperature, for $30 \mathrm{~min}$ to maintain identical conditions for each measurement. A schematic of the process is shown in Figure 2.

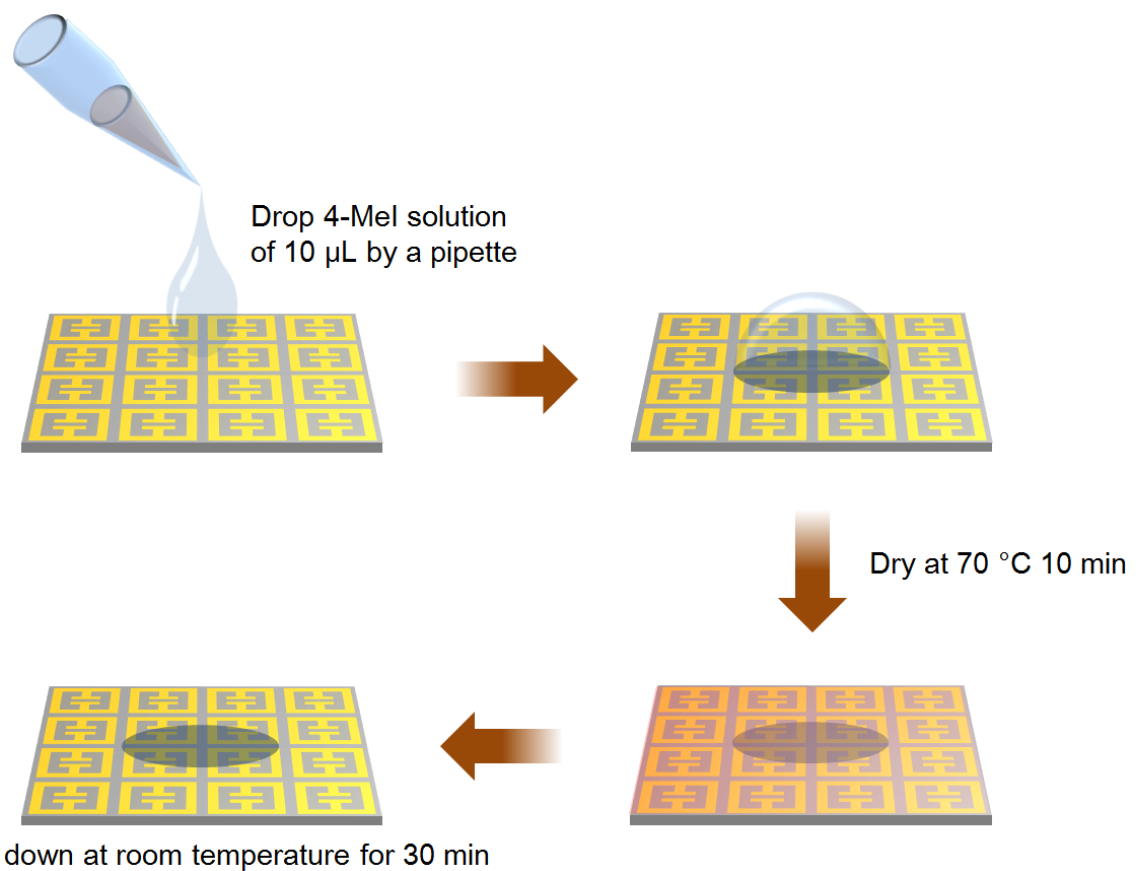

Figure 2. Schematic of the drop coating deposition of the 4-methylimidazole (4-MeI) sample on the $\mathrm{THz}$ metamaterial. 


\section{Results and Discussion}

\section{1. $\mathrm{THz}$ Spectrum of a 4-MeI Pellet}

We first described the THz spectral characteristics of the 4-MeI, using THz-TDS. We measured the THz spectra of a pellet composed of PE and 4-MeI, and the spectra are shown in Figure 3. As can be seen in the figure, the PE pellet showed a low absorption and had no resonance peak at the measured $\mathrm{THz}$ region. From the THz spectrum of the PE pellet, we obtained the THz spectrum of 4-MeI, by extracting the absorbance of the PE. The spectrum of the pellet composed of PE and the 4-MeI showed two significant 4-MeI resonance peaks at 0.56 and $0.82 \mathrm{THz}$. In particular, the spectrum of 4-MeI showed a more intense absorption peak at $0.82 \mathrm{THz}$ than at $0.56 \mathrm{THz}$.

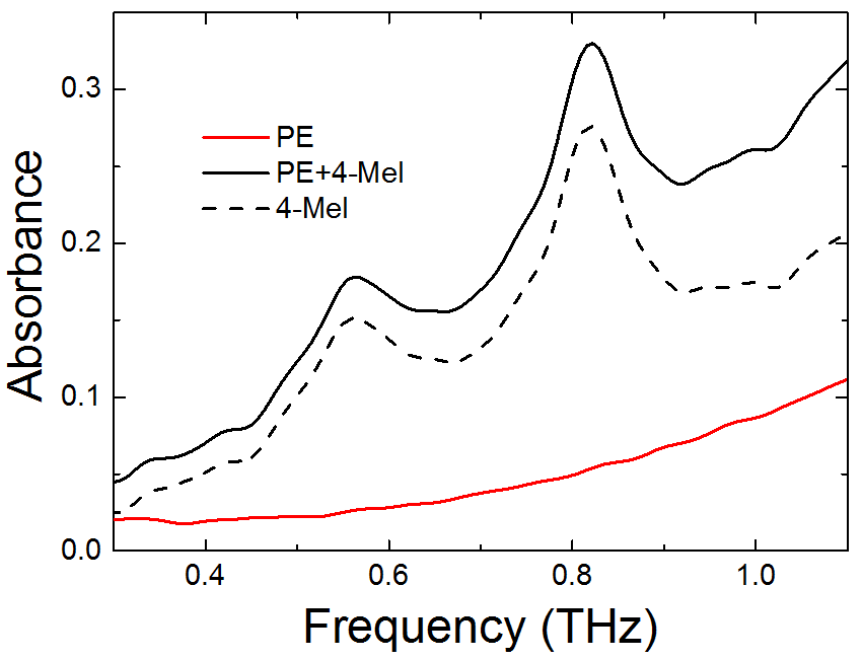

Figure 3. The spectra of polyethylene and 4-methylimidazole pellets at the THz region of 0.2 to $1.1 \mathrm{THz}$.

\subsection{4-MeI Detection in the THz Metamaterial}

To fabricate frequency-selective metamaterials for 4-MeI, as shown in Figure 1, the metamaterial was designed to encompass a resonance frequency of $0.82 \mathrm{THz}$, which was the resonance frequency of the 4-MeI. The resonant peak position of the transmittance was determined, based on the capacitor length and the gap of the metamaterial [31]. The capacitor length (C) and the gap (g) were designed to be $20 \mu \mathrm{m}$ and $8 \mu \mathrm{m}$. The full length (L) of the metamaterial was $54 \mu \mathrm{m}$, as shown in Figure $1 \mathrm{a}$. The simulation and experimental results are shown in Figure 4a. In the FDTD simulation, gold is considered to be a perfect electric conductor (PEC) and the relative permittivity of the PI layer was 2.88-0.09i. The normalized $\mathrm{THz}$ spectrum of the metamaterial illustrated a resonant feature at $0.82 \mathrm{THz}$. As can be seen in Figure 4a, the experimental spectrum of the THz metamaterial was in good agreement with the FDTD simulation. In addition, at the resonance frequency, we found that the localized $\mathrm{THz}$ electric field was strongly enhanced at the gap of the antenna, in the simulation, as shown in the inset, in Figure $4 \mathrm{a}$. Figure $4 \mathrm{~b}$ shows a scanning optical microscope image of the fabricated $\mathrm{THz}$ metamaterial. When the 4-MeI sample was dropped onto the $\mathrm{THz}$ metamaterial, the 4-MeI molecules significantly interacted with the localized strong THz field and the features of the metamaterial changed as the 4-MeI concentration increased. Consequently, the resonant peak position and the transmittance were changed. 


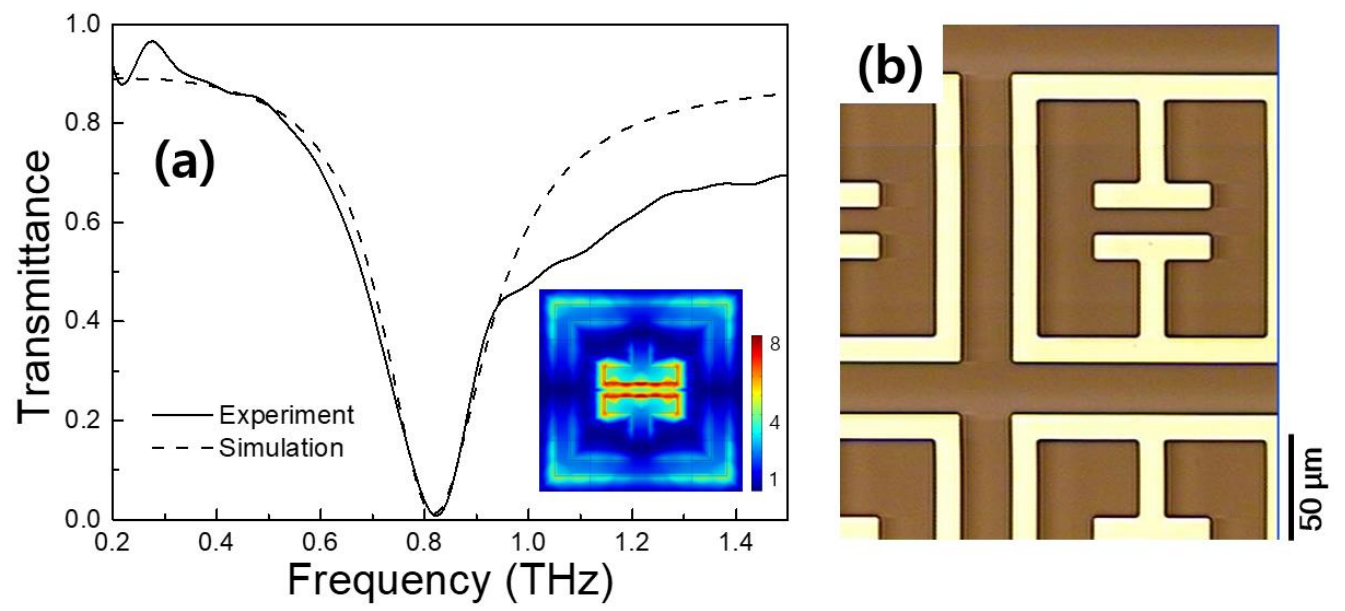

Figure 4. (a) Transmittance curves obtained in the experiment and the simulation, using the $\mathrm{THz}$ metamaterial. (b) Optical microscope image of the THz metamaterial.

Figure 5 illustrates the full $\mathrm{THz}$ spectra of the metamaterial, at different 4-MeI concentrations of $0,1,2,5,10,15$, and $20 \mathrm{mg} / \mathrm{L}$. The inset in Figure 5 shows a zoomed-in image of the resonant region. As seen in the figure, the resonant peak of the $\mathrm{THz}$ metamaterial shifted to a lower frequency as the 4-MeI concentration increased. In addition, the transmittance also increased slightly. The 4-MeI concentration-dependence of the resonance frequency and the transmittance of the $\mathrm{THz}$ metamaterial is shown in Figure 6. In Figure 6a, the resonance frequency was shifted to a lower frequency as the concentration of the 4-MeI increased. In addition, the transmittance increased as the concentration of the 4-MeI increased. The behavior of the resonance frequency and the transmittance could be explained by fitting to a linear equation. The resonance frequency shift-fitted to the linear equation $Y=-0.31 X+820.56$ and the transmittance change-fitted to $Y=8.1 \times 10^{-3} X+7.8 \times 10^{3}$, with increasing 4-MeI concentrations. As a result, the resonance frequency was shifted, by approximately $8 \mathrm{GHz}$, and the transmittance at the resonance frequency increased to $2 \times 10^{-3}$ as the concentration increased to $20 \mathrm{mg} / \mathrm{L}$. These results indicated that a few $\mathrm{mg} / \mathrm{L}$ of the $4-\mathrm{MeI}$ present in food materials, such as a beverage, can be detected using this metamaterial. However, the sensitivity of the metamaterial needs to be improved by using a higher sharp resonant peak or enhancing the interactions between the over-layered materials and an electric field near the metal structure, which would enable detection of 4-MeI of less than a few hundred $\mathrm{mg} / \mathrm{L}$.

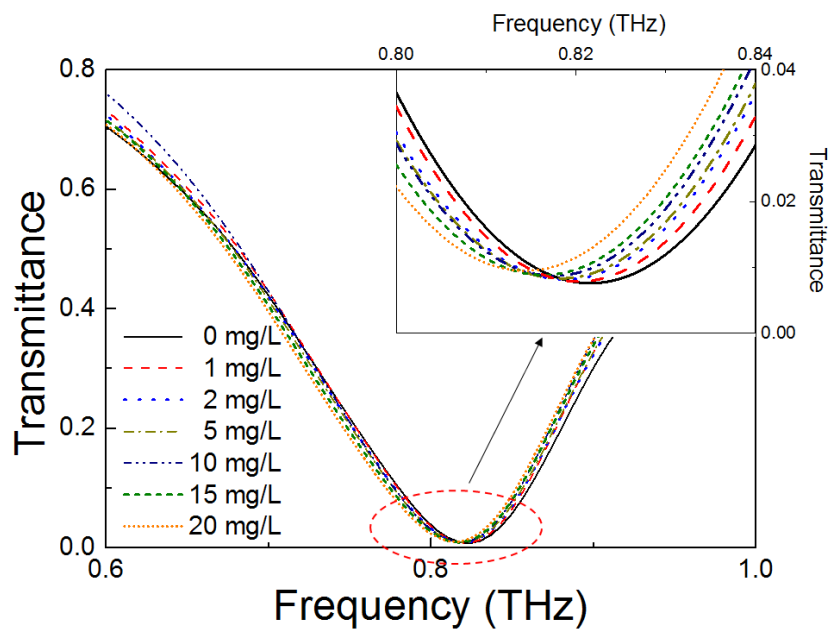

Figure 5. Spectra of the 4-MeI detection, using the THz metamaterial, at concentrations of $0,1,2,5,10$, 15 , and $20 \mathrm{mg} / \mathrm{L}$ in the $\mathrm{THz}$ region of 0.6 to $1.0 \mathrm{THz}$. 

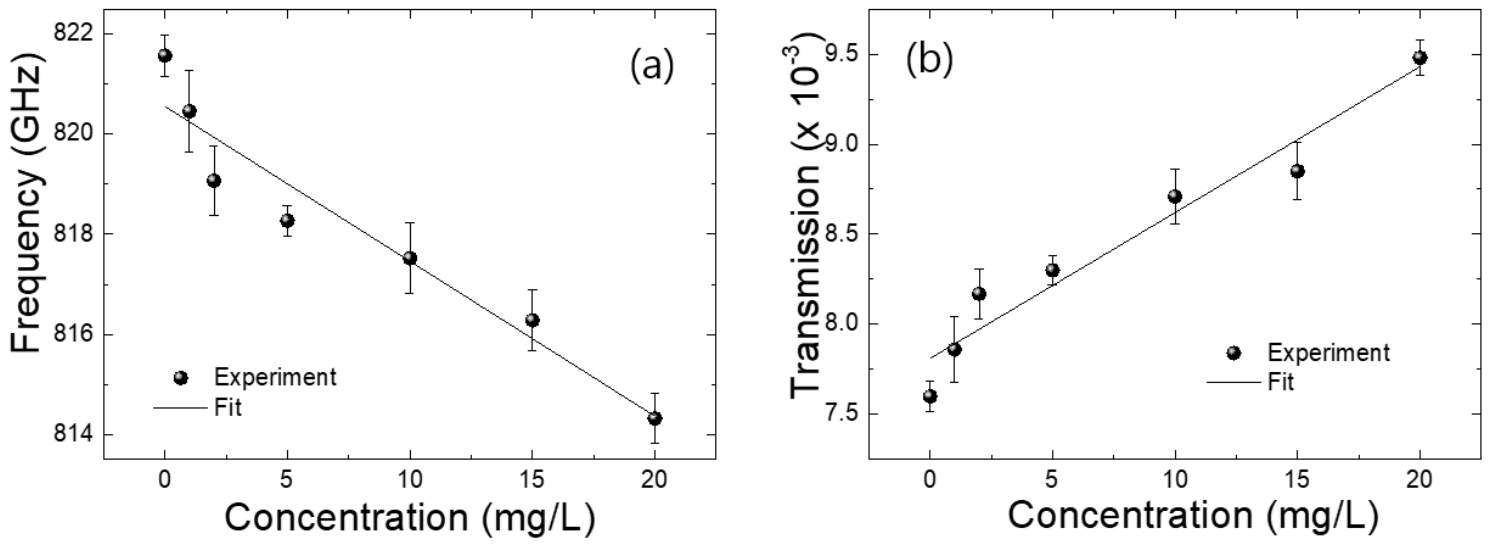

Figure 6. (a) The resonance frequency and (b) transmittance of the $\mathrm{THz}$ metamaterial as the concentration of 4-MeI increases. Solid lines indicate linear fits of the experimental results. All experimental results have been obtained through four different times of measurement.

The present study provided experimental evidence supporting the fact that the application of metamaterials, combined with the $\mathrm{THz}$ technology, has a substantial potential in the detection of hazardous materials in food products. Further studies may involve fabrication of the $\mathrm{THz}$ metamaterials with sharp resonant peaks and a high sensitivity, with a nano-slit structure, to improve the sensitivity of the metamaterials in detecting 4-MeI, as well as other carcinogenic substances.

\section{Conclusions}

In conclusion, we reported a $\mathrm{THz}$ metamaterial-based detection of the carcinogenic substance 4-MeI, which may be present in caramel-colored food products. We measured the $\mathrm{THz}$ spectra of pellets containing 4-MeI and PE and observed the resonance peaks at 0.56 and $0.82 \mathrm{THz}$. We fabricated a THz metamaterial, with a resonance feature at $0.82 \mathrm{THz}$, and measured the $\mathrm{THz}$ spectra at 4-MeI concentrations ranging from 0 to $20 \mathrm{mg} / \mathrm{L}$. We found that the resonance frequency decreased and the transmittance increased as the 4-MeI concentration increased. We were able to clearly detect 4-MeI, using the $\mathrm{THz}$ metamaterial, and the results demonstrated a highly sensitive detection. Thus, our findings provide new insight into 4-MeI detection and the potential applications of metamaterials-based technology, in food safety inspection.

Author Contributions: G.O. and H.J.S. conceived and designed the concept of the experiments. H.J.S. wrote the manuscript and analyzed the experimental results. 4-methylimidazole samples were prepared by H.W.J.. THz time-domain spectroscopy characteristics of 4-methylimidazole and metamaterials were carried out by H.J.S. All the authors participated in discussion and the results.

Funding: This work was supported financially by Main Research Program of the Korea Food Research Institute funded by the Ministry of Science, ICT \& Future Planning (E0187301-01).

Conflicts of Interest: The authors declare no conflict of interest.

\section{References}

1. Lee, K.G.; Jang, H.; Shibamoto, T. Formation of carcinogenic 4 (5)-methylimidazole in caramel model systems: A role of sulphite. Food Chem. 2013, 136, 1165-1168. [CrossRef] [PubMed]

2. Chappel, C.I.; Howell, J.C. Caramel colours-A historical introduction. Food Chem. Toxicol. 1992, 30, 351-357. [CrossRef]

3. Yamaguchi, H.; Masuda, T. Determination of 4-MI in soy sauce and other foods by LC-MS after solid-phase extraction. J. Agric. Food Chem. 2011, 59, 9770-9775. [CrossRef] [PubMed]

4. Hengel, M.; Shibamoto, T. Carcinogenic 4 (5)-methylimidazole found in beverages, sauces, and caramel colors: Chemical properties, analysis, and biological activities. J. Agric. Food Chem. 2013, 61, 780-789. [CrossRef] 
5. Moon, J.K.; Shibamoto, T. Formation of carcinogenic 4 (5)-methylimidazole in Maillard reaction systems. J. Agric. Food Chem. 2010, 59, 615-618. [CrossRef] [PubMed]

6. Lawrence, J.F.; Charbonneau, C.F. Direct method for the determination of 2-acetyl-4 (5)-tetrahydroxybutylimidazole in caramel colours and beers by high-performance liquid chromatography. J. Chromatogr. A 1987, 407, 405-407. [CrossRef]

7. Klejdus, B.; Moravcová, J.; Kubáň, V. Reversed-phase high-performance liquid chromatographic/mass spectrometric method for separation of 4-methylimidazole and 2-acetyl-4-(1,2,3,4-tetrahydroxybutyl) imidazole at pg levels. Anal. Chim. Acta 2003, 477, 49-58. [CrossRef]

8. Cerny, M.; Blumenthal, A. 4-Methylimidazol in Caramel und caramelgefärbten Lebensmitteln. Z. Lebensm. Unters. Forsch. A 1979, 168, 87-90. [CrossRef]

9. Fuchs, G.; Sundell, S. Quantitative determination of 4-methylimidazole as 1-acetyl derivative in caramel color by gas-liquid chromatography. J. Agric. Food Chem. 1975, 23, 120-122. [CrossRef]

10. Pons, I.; Garrault, C.; Jaubert, J.N.; Morel, J.; Fenyo, J.C. Analysis of aromatic caramel. Food Chem. 1991, 39, 311-320. [CrossRef]

11. Liu, J.; Sun, J.; Sui, X.; Wang, Y.; Hou, Y.; He, Z. Predicting blood-brain barrier penetration of drugs by microemulsion liquid chromatography with corrected retention factor. J. Chromatogr. A 2008, 1198, 164-172. [CrossRef] [PubMed]

12. Moretton, C.; Crétier, G.; Nigay, H.; Rocca, J.L. Quantification of 4-methylimidazole in class III and IV caramel colors: Validation of a new method based on heart-cutting two-dimensional liquid chromatography (LC-LC). J. Agric. Food Chem. 2011, 59, 3544-3550. [CrossRef] [PubMed]

13. Matthews, H.R.; Rapoport, H. Differentiation of 1, 4-and 1, 5-disubstituted imidazoles. J. Am. Chem. Soc. 1973, 95, 2297-2303. [CrossRef]

14. Bowie, J.H.; Cooks, R.G.; Lawesson, S.; Schroll, G. Electron impact studies. XII. Mass spectra of substituted imidazoles. Aust. J. Chem. 1967, 20, 1613-1624. [CrossRef]

15. Smye, S.W.; Chamberlain, J.M.; Fitzgerald, A.J.; Berry, E. The interaction between terahertz radiation and biological tissue. Phys. Med. Biol. 2001, 46, R101. [CrossRef] [PubMed]

16. Upadhya, P.C.; Shen, Y.C.; Davies, A.G.; Linfield, E.H. Terahertz time-domain spectroscopy of glucose and uric acid. J. Biol. Phys. 2003, 29, 117-121. [CrossRef] [PubMed]

17. Ajito, K.; Ueno, Y. THz chemical imaging for biological applications. IEEE Trans. Terahertz Sci. Technol. 2011, 1, 293-300. [CrossRef]

18. Shin, H.J.; Oh, S.J.; Kim, S.I.; Won Kim, H.; Son, J.H. Conformational characteristics of $\beta$-glucan in laminarin probed by terahertz spectroscopy. Appl. Phys. Lett. 2009, 94, 111911. [CrossRef]

19. Otsuji, T.; Popov, V.; Ryzhii, V. Active graphene plasmonics for terahertz device applications. J. Phys. D Appl. Phys. 2014, 47, 094006. [CrossRef]

20. Shin, H.J.; Kim, J.; Kim, S.; Choi, H.; Lee, S.; Lee, Y.H.; Son, J.H.; Lim, S.C. Unsaturated drift velocity of monolayer graphene. Nano Lett. 2018, 18, 1575-1581. [CrossRef]

21. Shin, H.J.; Kim, S.H.; Park, K.; Lim, M.C.; Choi, S.W.; Ok, G. Free-standing guided-mode resonance humidity sensor in terahertz. Sens. Actuators A Phys. 2017, 268, 27-31. [CrossRef]

22. Mayorga, I.C.; Schmitz, A.; Klein, T.; Leinz, C.; Gusten, R. First in-field application of a full photonic local oscillator to terahertz astronomy. IEEE Trans. Terahertz Sci. Technol. 2012, 2, 393-399. [CrossRef]

23. Ergün, S.; Sönmez, S. Terahertz technology for military applications. J. Manag. Inf. Sci. 2015, 3, 13-16. [CrossRef]

24. Shin, H.J.; Choi, S.W.; Ok, G. Qualitative identification of food materials by complex refractive index mapping in the terahertz range. Food Chem. 2018, 245, 282-288. [CrossRef] [PubMed]

25. Ok, G.; Park, K.; Lim, M.C.; Jang, H.J.; Choi, S.W. 140-GHz subwavelength transmission imaging for foreign body inspection in food products. J. Food Eng. 2018, 221, 124-131. [CrossRef]

26. Ji, Y.B.; Moon, I.S.; Bark, H.S.; Kim, S.H.; Park, D.W.; Noh, S.K.; Jeon, T.I. Terahertz otoscope and potential for diagnosing otitis media. Biomed. Opt. Express 2016, 7, 1201. [CrossRef] [PubMed]

27. Oh, S.J.; Kim, S.H.; Ji, Y.B.; Jeong, K.; Park, Y.; Yang, J.; Park, D.W.; Noh, S.K.; Kang, S.G.; Huh, Y.M.; et al. Study of freshly excised brain tissues using terahertz imaging. Biomed. Opt. Express 2014, 5, 2837-2842. [CrossRef]

28. Zhong, H.; Redo-Sanchez, A.; Zhang, X.C. Identification and classification of chemicals using terahertz reflective spectroscopic focal-plane imaging system. Opt. Express 2006, 14, 9130-9141. [CrossRef] 
29. Walther, M.; Fischer, B.M.; Ortner, A.; Bitzer, A.; Thoman, A.; Helm, H. Chemical sensing and imaging with pulsed terahertz radiation. Anal. Bioanal. Chem. 2010, 397, 1009-1017. [CrossRef]

30. Rungsawang, R.; Ueno, Y.; Tomita, I.; Ajito, K. Terahertz notch filter using intermolecular hydrogen bonds in a sucrose crystal. Opt. Express 2006, 14, 5765-5772. [CrossRef]

31. Park, S.J.; Hong, J.T.; Choi, S.J.; Kim, H.S.; Park, W.K.; Han, S.T.; Park, J.Y.; Lee, S.; Kim, D.S.; Ahn, Y.H. Detection of microorganisms using terahertz metamaterials. Sci. Rep. 2014, 4, 1-7. [CrossRef] [PubMed]

32. Reinhard, B.; Schmitt, K.M.; Wollrab, V.; Neu, J.; Beigang, R.; Rahm, M. Metamaterial near-field sensor for deep-subwavelength thickness measurements and sensitive refractometry in the terahertz frequency range. Appl. Phys. Lett. 2012, 100, 221101. [CrossRef]

33. Tao, H.; Strikwerda, A.C.; Liu, M.; Mondia, J.P.; Ekmekci, E.; Fan, K.; Kaplan, D.L.; Padilla, W.J.; Zhang, X.; Averitt, R.D.; et al. Performance enhancement of terahertz metamaterials on ultrathin substrates for sensing applications. Appl. Phys. Lett. 2010, 97, 261909. [CrossRef]

34. Xu, W.; Xie, L.; Zhu, J.; Wang, W.; Ye, Z.; Ma, Y.; Tsai, C.Y.; Chen, S.; Ying, Y. Terahertz sensing of chlorpyrifos-methyl using metamaterials. Food Chem. 2017, 218, 330-334. [CrossRef] [PubMed]

35. Qin, J.; Xie, L.; Ying, Y. A high-sensitivity terahertz spectroscopy technology for tetracycline hydrochloride detection using metamaterials. Food Chem. 2016, 211, 300-305. [CrossRef] [PubMed]

36. O'Hara, J.F.; Singh, R.; Brener, I.; Smirnova, E.; Han, J.; Taylor, A.J.; Zhang, W. Thin-film sensing with planar terahertz metamaterials: sensitivity and limitations. Opt. Express 2008, 16, 1786-1795. [CrossRef] [PubMed]

37. Singh, R.; Cao, W.; Al-Naib, I.; Cong, L.; Withayachumnankul, W.; Zhang, W. Ultrasensitive terahertz sensing with high-Q Fano resonances in metasurfaces. Appl. Phys. Lett. 2014, 105, 171101. [CrossRef]

38. Shih, K.; Pitchappa, P.; Manjappa, M.; Ho, C.P.; Singh, R.; Lee, C. Microfluidic metamaterial sensor: Selective trapping and remote sensing of microparticles. J. Appl. Phys. 2017, 121, 023102. [CrossRef]

39. Serita, K.; Matsuda, E.; Okada, K.; Murakami, H.; Kawayama, I.; Tonouchi, M. Terahertz microfluidic chips sensitivity-enhanced with a few arrays of meta-atoms. APL Photonics 2018, 3, 051603. [CrossRef]

40. Schurig, D.; Mock, J.J.; Smith, D.R. Electric-field-coupled resonators for negative permittivity metamaterials. Appl. Phys. Lett. 2016, 88, 041109. [CrossRef]

41. Zhang, C.H.; Wu, J.B.; Jin, B.B.; Ji, Z.M.; Kang, L.; Xu, W.W.; Chen, J.; Tonouchi, M.; Wu, P.H. Low-loss terahertz metamaterial from superconducting niobium nitride films. Opt. Express 2012, 20, 42-47. [CrossRef] [PubMed] 